\section{Integração Externa e Capacidade de Inovação Organizacional Moderada pelo Nível de Confiança}

\author{
Sidnei Gripa e Luciano Castro de Carvalho
}

\section{RESUMO}

A integração da cadeia de suprimentos (ICS) possibilita vantagem competitiva no que diz respeito ao desempenho operacional. Isso já está consolidado na literatura. A lacuna existente está relacioanada com a ICS e a capacidade de inovação das organizações, pois de acordo com vários autores, ainda não há dados suficientes que comprovem essa relação. Este estudo tem como objetivo identificar se integração de clientes e fornecedores influenciam a capacidade de inovação organizacional. Para isso, foi realizada uma pesquisa descritiva com método quantitativo, utilizando-se de dados primários provindos de um survey. Os dados foram coletados por meio de um questionário on-line, com 20 questões, contendo dimensões sobre envolvimento de clientes e fornecedores no processo de desenvolvimento de produto, confiança e capacidade de inovação. Foram coletados dados de 86 empresas da indústria de transformação de portes variados. Os dados foram analisados por modelagem de equações estruturais com utilização do software SmartPLS. Os resultados indicam que o envolvimento do cliente influencia 0,52 na capacidade de inovação organizacional, porém o envolvimento do fornecedor, bem como a moderação da confiança sobre essas relações não foram significativas. Conclui-se que a integração da cadeia de suprimentos, principalmente com o envolvimento dos clientes, influencia a capacidade de inovação.

Palavras-chave: Integração da cadeia de suprimentos; Capacidade de inovação; Confiança

\section{External Integration and Organizational Innovation Capacity Moderate by The Confidence Level}

\section{ABSTRACT}

Supply chain integration allows competitive advantage with respect to the operational performance for all those involved in this process. This is already well consolidated in the literature. The existing gap is related to the integration of the supply chain and the innovation capacity of organizations, since according to several authors, there still isn't enough data to prove this relationship. This study aims to identify whether integration of customers and suppliers influence on the organizational innovation capacity. For this, a descriptive research with quantitative method was carried out, using primary data from a survey. The data were collected through an online questionnaire with 20 questions, containing dimensions on customer and supplier involvement in product development process, trust and innovation capacity. Data were collected from 86 companies of the processing industry of varying sizes. The data were analyzed by structural equation modelling using SmartPLS software. The results indicate that the customer engagement influences 0.52 on organizational innovation capacity, but the involvement with suppliers, as well as moderation of trust about these relationships were not significant. It is concluded that the supply chain integration, mainly with the involvement of the costumers, influences the innovation capacity.

Keywords: Supply chain integration; Innovation capacity; Trust.

Recebido em: 15/12/2017

Aprovado em: 24/07/2018 Última Modificação: 13/09/2018

Check for updates

Sidnei Gripa (iD), Coordenador dos cursos de Administração, Gestão Comercial, Logística e Processos Gerenciais no Centro Universitário de Brusque, Brasil. Doutorando em Ciências Contábeis e Administração pela Fundação Universidade Regional de Blumenau, Brasil.

gripa@unifebe.edu.br

Luciano Castro de Carvalho (iD,

Programa de Pós-graduação em Ciências Contábeis e Administração, Fundação Universidade Regional de Blumenau, Brasil.

Doutor em Administração pela

Fundação Getúlio Vargas, São Paulo, Brasil

luccar@gmail.com 
Introdução

Para enfrentar a competição global, as organizações estão deixando de atuar de forma isolada para atuar de maneira conjunta com outras organizações da cadeia de suprimentos (CS). A transição da eficiência individual para a eficiência coletiva é uma das principais características da nova economia (Bornia \& Lorandi, 2016). A transição da atuação individual para atuação coletiva necessita de uma mudança na CS, que deverá ser voltada para o aumento do desempenho coletivo e não somente individual (Bornia \& Lorandi, 2016). No intuito de obter e sustentar os recursos necessários, as organizações devem mudar sua estrutura e comportamento e formar alianças que promovam benefícios mútuos (Zhao, Huo, Flynn \& Yeung, 2008; Lii \& Kuo, 2016).

Os benefcios da integração da cadeia de suprimentos podem ser alcançados por meio de uma ligação eficiente entre várias atividades da CS, tais como: a aquisição de matéria-prima, processo de produção, distribuição etc. Essa ligação entre organizações deve estar sujeita à construção e utilização efetiva de várias práticas, tais como: responsabilidade, confiabilidade, transparência, entre outras, para gerar uma cadeia de suprimentos integrada (Kim, 2009). Assim, o alto desempenho da cadeia de suprimentos só pode ser alcançado quando as organizações integram suas operações com fornecedores ou clientes (Zhao et al., 2008; Kim, 2009; Lau, Yam, \& Tang, 2010; Wong, Wong, \& Boon-Itt, 2013; Lii \& Kuo, 2016).

A gestão da CS possilita às organizações uma melhora na integração interna (II) e uma colaboração efetiva com fornecedores e clientes por meio da integração externa (IE) para aumentar sua competitividade (Kim, 2009; Wong, et al., 2013). Wong et al., (2013) reforçam que a integração da CS é amplamente aceita como tendo a capacidade de melhorar os resultados do desempenho operacional, a saber: qualidade, custo, entrega e flexibilidade.

Na integração externa, a confiança promove e fortalece a cooperação e a coordenação entre os parceiros da cadeia de suprimentos (Ojha, Shockley, \& Acharya, 2016). Quando os parceiros da SC estabelecem relações de confiança, conhecimentos, informações e outros recursos estratégicos são mais propensos de serem compartilhados de forma eficaz e colaborativa (Ojha et al., 2016). Este cenário colaborativo, com base na confiança entre parceiros, pode auxiliar na adaptação das organizações ao ambiente dinâmico de negócios e às mudanças no comportamento de compra dos consumidores. Para isso, as empresas devem lançar produtos ou serviços mais inovadores para conseguir atender às necessidades do mercado (Lii \& Kuo, 2016).

Dessa forma, verifica-se que a integração da cadeia de suprimentos possui relação positiva com o desempenho da empresa no que diz respeito aos aspectos estratégicos, organizacionais, de informação e de mercado. Apesar de os estudos mencionados sugerirem uma melhora nos resultados da organização, pouco se sabe se essa interação desenvolve ou aumenta a capacidade de inovação das empresas (Prajogo \& Olhager, 2012; Liu, Ke, Kee Wei, \& Hua, 2013; Wong et al., 2013; Lii \& Kuo, 2016). 
Assim, questiona-se se além do desempenho operacional obtido pelas organizações por meio da integração externa, se esta desenvolve ou aumenta a capacidade de inovação das organizações. Diante desse contexto, estabelece-se como pergunta de pesquisa: Qual a influência da integração com fornecedores e clientes na capacidade de inovação organizacional quando moderada pelo nível de confiança entre parceiros? Para responder à pergunta de pesquisa, delimitou-se como objetivo geral identificar se a integração de clientes e fornecedores influencia a capacidade de inovação organizacional.

As análises partem dos pressupostos da teoria da dependência de recursos (TDR), a qual fornece uma lente útil para a compreensão da integração da cadeia de suprimentos e a capacidade de inovação das empresas. De acordo com a TDR, é importante criar ambientes de negociação e estabelecer acordos interorganizacionais como respostas estratégicas às mudanças (Meznar \& Nigh, 1995; Buvik \& Gronhaug, 2000). Para Lii e Kuo (2016), a essência da teoria da dependência de recursos ocorre quando uma organização, que não pode gerar os recursos necessários, entra em uma relação de troca com outras organizações.

Este artigo está estruturado em cinco capítulos. Além desta introdução, no capítulo dois está o referencial teórico que dá suporte ao estudo. No capítulo três está detalhada a metodologia utilizada. O capítulo quatro contempla a análise dos resultados e as discussões. Por último, o capítulo cinco contempla as considerações finais.

\section{Deferencial teórico}

Apresentam-se nesta seção as referências sobre a teoria da dependência de recursos - TDR, a capacidade de inovação organizacional, a integração da cadeia de suprimentos e a confiança nas relações interorganizacionais. Estas referências são necessárias para o embasamento conceitual do problema de pesquisa, bem como a apresentação das hipóteses.

\section{Teoria da dependência de recursos - TDR}

A teoria da dependência de Recursos (TDR) é baseada em grande parte nas discussões de Pfeffer e Salancik (2003). A principal questão abordada pela Teoria da Dependência de Recursos refere-se a entender por que as organizações entram em arranjos interorganizacionais (Drees \& Heugens, 2013). O argumento básico da dependência dos recursos está no âmbito das organizações que são limitadas por uma rede de interdependências externa, levando a uma situação em que a sobrevivência e o sucesso são incertos. Assim, as organizações tomam ações para gerenciar as interdependências e geram novos padrões de dependência com efeito sobre o comportamento organizacional (Pfeffer,1987).

A Teoria da Dependência de Recursos tem como premissa básica a noção de que todas as organizações dependem criticamente de outras organizações para a provisão de recursos essenciais e que essa dependência é frequentemente recíproca entre as organizações (Drees \& Heugens, 2013). 
Incerteza e dependência são as duas principais variáveis discutidas pela TDR, cujas propriedades podem ser explicadas com base em uma descrição das características do ambiente e na relação de troca dos recursos no relacionamento em questão (Schiele, Ellis, Eßig, Henke \& Kull, 2015).

A formação de alianças cooperativas tem se mostrado como uma estratégia eficiente para facilitar 0 acesso das organizações a muitos recursos dos quais elas dependem. Nessa perspectiva, as empresas são vistas como organizações que formam colaborações interorganizacionais de diversas intensidades, a fim de adquirir e manter os recursos necessários para a sobrevivência (Schiele et al., 2015).

Apesar do seu potencial como uma das principais teorias para explicar as relações entre as organizações e o meio ambiente, a TDR ainda não é tão explorada e testada como poderia ser (Pfeffer \& Salancik, 2003). Nesse sentido, este estudo busca identificar, por meio da lente da TDR, a influência das relações interorganizacionais com fornecedores e clientes na capacidade de inovação da empresa focal.

\section{Capacidade de inovação organizacional e integração da cadeia de suprimentos}

O atual ambiente dinâmico obriga as organizações a buscarem continuamente a inovação para sustentar a vantagem competitiva (Ayoub, Abdallah \& Suifan, 2017). A necessidade das organizações de inovar pode gerar novos produtos, serviços, processos, tecnologia ou mesmo modelos de negócios, que agregam valor aos clientes (Skippari, Laukkanen, \& Salo, 2017).

A inovação de produto é orientada principalmente pelas necessidades do cliente e do mercado, e tem como objetivo desenvolver novos produtos e melhorar a qualidade deles. (Birasnav, Albufalasa, \& Bader, 2013; Ayoub, et al. 2017). Já a inovação de processos orienta-se pelas necessidades da manufatura e tem como objetivo minimizar os custos de produção (Ayoub, et al. 2017). Inovação é um processo interativo iniciado pela percepção de um novo mercado e/ou nova oportunidade de serviço para uma invenção baseada na tecnologia que leva a tarefas de desenvolvimento, produção e comercialização que se esforçam para o sucesso comercial da invenção (MANUAL, 2005).

A infraestrutura estratégica necessária para desenvolver a inovação na cadeia de suprimentos tornou-se uma área de interesse dos profissionais e de toda a cadeia de suprimentos (Ojha et al., 2016). Essa tendência está transformando os parceiros externos em uma fonte cada vez mais importante de inovação de produtos e processos (Jean, Kim \& Sinkovics, 2012). Segundo Szeto (2000), a capacidade de inovação pode ser definida como os recursos necessários para transformar oportunidades em novos produtos e processos e atender às necessidades dos clientes. Para Van de Ven (1986), a inovação exige uma quantidade substancial de recursos que pode exceder a capacidade de algumas empresas.

A capacidade de inovação permite que as empresas percebam oportunidades e ameaças, tomem decisões oportunas, orientadas para o cliente e reconfigurarem os recursos de rede para atender às expectativas 
crescentes dos clientes (Fawcett, Jones, \& Fawcett, 2012). A capacidade de inovação é considerada ponto chave para o sucesso das empresas em cenário de competitividade global. Assim, a capacidade de inovação no relacionamento com parceiros da cadeia de suprimentos refere-se à aplicação ou utilização de recursos externos para gerar novos produtos ou processos em relações de cooperação e troca (Jean et al., 2012).

A integração do cliente acontece na medida em que os clientes e os fabricantes coordenam conjuntamente as decisões relacionadas ao nível de estoque, planejamento de produção, previsão de demanda, acompanhamento de pedidos, entre outras atividades relacionadas à gestão do negócio (Wong, Boon-Itt ,\& Wong, 2011; He, Lai, Sun \& Chen, 2014). Voss (2012) sugere em seus estudos que os clientes devem ser considerados nas decisões sobre as quais os projetos devam ser priorizados, adicionados ou retirados da carteira, e como alocar recursos entre os projetos.

Em uma pesquisa realizada com 18 gestores de empresas suecas e norueguesas, Stenmark, Tinnsten e Wiklund (2011) afirmam que os clientes são as fontes de informação mais importantes no desenvolvimento de ideias. Treze empresas, ou seja, 72\% disseram utilizar feedback de seus clientes na fase de uso dos produtos e quinze empresas disseram que iriam aumentar o envolvimento de seus clientes no processo de desenvolvimento de novos produtos (DNP), tamanha a importância do cliente nessa etapa.

O envolvimento dos clientes apresenta relação positiva com a inovação do produto e o desempenho do novo produto (Abdolmaleki \& Ahmadian, 2016). Para Johnson e Filippini (2009), o envolvimento do cliente no DNP também pode acelerar o processo de desenvolvimento, levando a um melhor desempenho das organizações.

Uma organização que integra o cliente tende a melhorar a criatividade, produzindo novos e significativos produtos e processos que, por sua vez, aumentam seu potencial de inovação por meio de todo o seu sistema de negócios (Jean et al., 2012).

Esse processo se dá a partir das relações estreitas do marketing com os clientes para identificar suas preferências e depois coordenar com a P\&D o projeto dos produtos e processos (Gonzalez-Zapatero, Gonzalez-Benito, \& Lannelongue, 2017). Além disso, a integração com os clientes aprimora o compartilhamento de informações em todas as fases do desenvolvimento de novos produtos, gerando, assim, maior capacidade de inovação (Ayoub, et al., 2017). Dessa forma, tem-se que:

H1: A integração externa com os clientes está positivamente relacionada com a capacidade de inovação organizacional.

Sob outra perspectiva, o envolvimento com fornecedores pode trazer vantagem significativa para o processo de desenvolvimento do produto $(\mathrm{He}$ et al., 2014). Os fornecedores geralmente têm maior conhecimento sobre as especificações, peças e componentes que podem ser essenciais para o desenvolvimento de novos produtos (Sun, Yau, Suen, \& Kwok, 2010). 
O envolvimento precoce dos fornecedores pode melhorar o tempo de comercialização, qualidade, inovação tecnológica de novos produtos (Handfield, Ragatz, Petersen, \& Monczka, 1999) e velocidade de desenvolvimento de novos produtos (Langerak \& Hultink, 2008). Com dados de mais de 600 fabricantes em 21 países, Sun et al., (2010) afirmam que a integração com o fornecedor influencia positivamente o desempenho dos novos produtos, incluindo qualidade, confiabilidade, inovação e tempo de mercado.

Após avaliar 251 fabricantes de Hong Kong, Lau et al., (2010) encontraram uma relação direta e positiva entre a integração do fornecedor, do cliente e o desempenho do produto. Em particular, esse estudo verificou que o compartilhamento de informações com os fornecedores e o desenvolvimento de produtos com os clientes melhora diretamente o desempenho do produto (Lau et al., 2010).

O envolvimento dos fornecedores no processo de desenvolvimento de novos produtos permite a utilização das melhores peças e matérias-primas por parte dos fabricantes, facilitando a concepção dos produtos e minimizando o tempo do projeto (Ayoub et al., , 2017). Além disso, a integração com fornecedores aumenta a transparência das informações complexas sobre o produto e o processo de produção (Shou, Li, Park, \& Kang, 2017).

A criação conjunta de senso comum entre a organização e os parceiros externos possibilitam avanço na capacidade de inovação (Jean et al., 2012). De acordo com Jean et al. (2012), as partes interpretam a informação em conjunto por equipes cruzadas e reuniões frequentes, as quais permitem que as empresas cheguem a um consenso mais eficiente e evitem conflito na relação de troca. Somente por meio de senso comum as organizações podem filtrar as informações que recebem dos fornecedores, transformando-as em produtos com fins comerciais, potencializando a capacidade de inovação organizacional. Portanto, tem-se que:

H2: A integração externa com os fornecedores está positivamente relacionada com a capacidade de inovação organizacional.

\section{Confiança nas relações interorganizacionais}

A confiança na cadeia de suprimentos é uma relação de mão dupla entre os parceiros, o que implica alto nível de fé na decência dos outros, bem como no cumprimento das promessas sem afetar negativamente o parceiro (Chang, Ouzrout, Nongaillard, Bouras, \& Jiliu, 2014). Em outras palavras, quando há confiança entre parceiros, calcula-se a probabilidade de que vão agir sem oportunismo na parceria (He et al., 2014).

A confiança é um mecanismo de relação de governança que pode gerar resultados econômicos sobrenaturais para as organizações da cadeia de suprimentos que trabalham de forma integrada, uma vez que a confiança mútua impede o comportamento oportunista unilateral (Yeniyurt, Henke Jr., \& Yalcinkaya, 2014). Embora a TDR não aborde especificamente os mecanismos de governança, ela pontua que a dependência é a variável central que leva as firmas a estruturarem relacionamentos com outras 
firmas (Martins, 2013). Assim, de acordo com Martins (2013), a governança interfirma é uma resposta estratégica para a redução da dependência, a partir da estruturação das relações por elos formais e informais.

A ausência de confiança entre os membros da cadeia de suprimentos pode dificultar as relações e os resultados podem ser menos satisfatórios para os envolvidos (He et al., 2014). Do lado oposto, o compartilhamento de informações, o compromisso e a confiança entre os membros da cadeia de suprimentos tornaram-se elementos essenciais para a implementação bem-sucedida da ICS (Vijayasarathy, 2010).

Com objetivo de investigar o papel do compartilhamento de informações, qualidade da informação e disponibilidade de informações no desenvolvimento de confiança e compromisso nas relações da SC de organizações Canadenses e Taiwanesas, Chen, Yen, Rajkumar e Tomochko (2011) descobriram que a qualidade e a disponibilidade da informação desempenham um papel positivo na construção de confiança e de compromisso na SC.

O modelo de Handfield e Bechtel (2002), testado com dados coletados de uma amostra de gerentes de compras em empresas de manufatura norte-americanas, sugere que a dependência do comprador, os investimentos em ativos humanos dos fornecedores e a confiança estão positivamente associados com a capacidade de resposta da cadeia de suprimento, definida neste estudo como a capacidade de o fornecedor responder rapidamente às necessidades da parte compradora. Os resultados sugerem que mesmo nos casos em que os compradores não têm um grande controle sobre seus fornecedores, trabalhar para construir confiança dentro do relacionamento pode melhorar a capacidade de resposta do fornecedor.

Relacionando os impactos positivos e negativos da confiança na capacidade de inovação de 156 empresas em distritos industriais, MolinaMorales, Martínez-Fernández e Torlò (2011) concluem que alguns níveis de confiança são benéficos porque permitem a transferência de conhecimento tácito, mas as empresas que investem muito na confiança, assumindo riscos desnecessários, podem ter efeitos negativos na capacidade de inovação.

Em um estudo com 90 trios de organizações interligadas na SC em três países europeus, Kühne, Gellynck e Weaver (2013) identificaram três níveis de capacidade de inovação: alto, médio e baixo. A qualidade do relacionamento é definida por meio de características como confiança, satisfação social, poder não coercitivo e reputação. Os resultados sugerem que as características da qualidade da relação de cadeia podem ser fatores importantes para a melhoria da capacidade de inovação das organizações.

Uma característica chave do envolvimento externo dos parceiros em geral e, especificamente para a inovação, é a existência da confiança, pois ela reduz os custos e riscos envolvidos na colaboração entre os agentes da cadeia de suprimentos, ao mesmo tempo em que aumenta desempenho (Bunduchi, 2013). Envolver clientes e fornecedores nos processos organizacionais sugere o entendimento de como administrar os relacionamentos por meio da confiança, que é um importante passo para o discurso da integração de clientes e fornecedores nos processos de desenvolvimento de produtos ( $\mathrm{He}$ et al., 2014). Com esse entendimento tem-se que: 
$\mathrm{H}_{3 \mathrm{a}}$ : A confiança modera a relação entre o envolvimento do cliente e a capacidade de inovação.

$H_{3 b}$ : A confiança modera a relação entre o envolvimento do fornecedor e a capacidade de inovação.

Com base no exposto e nas hipóteses geradas, a Figura 1 apresenta o modelo teórico de pesquisa:

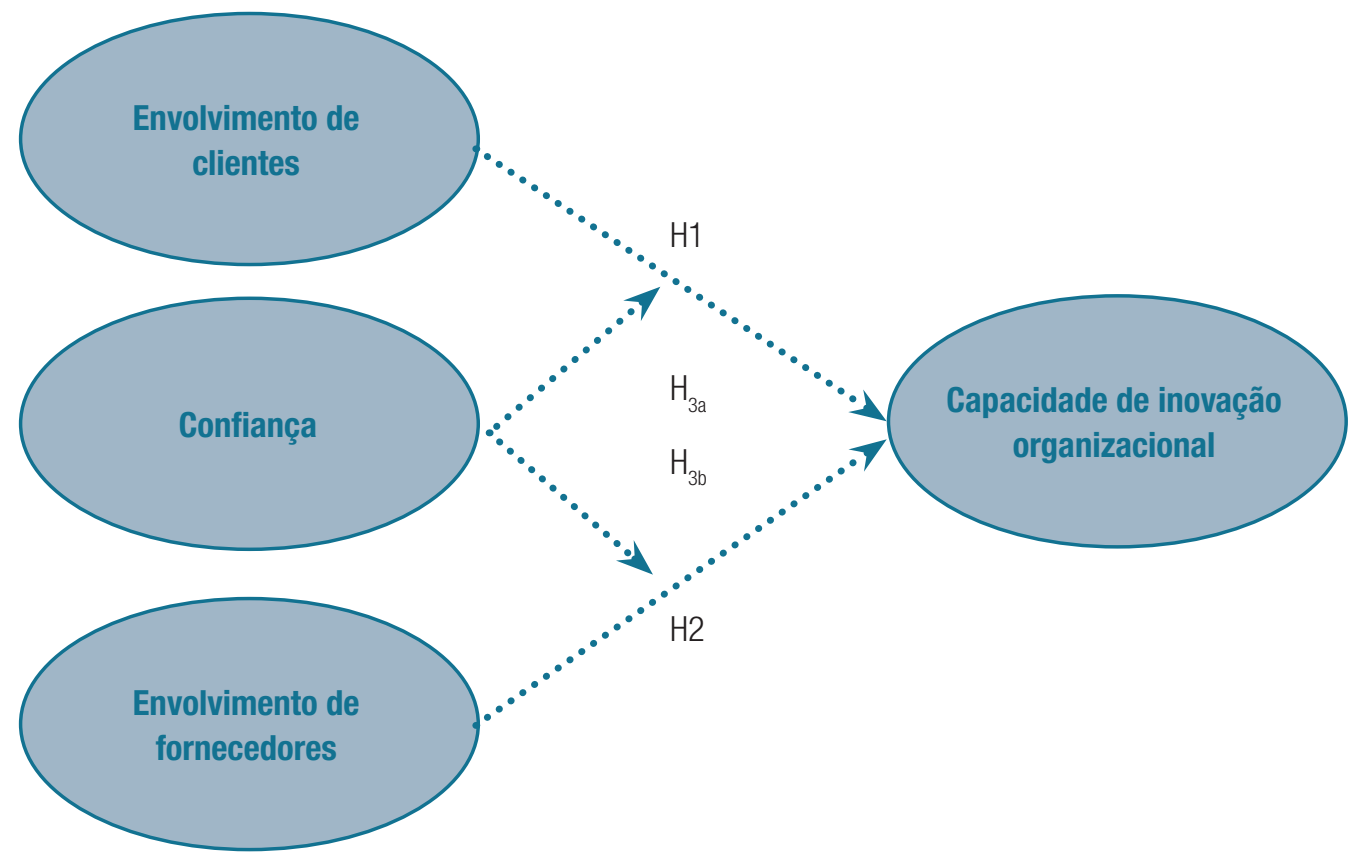

Figura 1: Modelo teórico

Fonte: dados da pesquisa

\section{Metodologia de pesquisa}

O método utilizado para atingir o objetivo proposto pode ser classificado como uma pesquisa quantitativa de natureza descritiva, utilizando-se de dados primários provindos de um survey. A amostra utilizada neste estudo foi a não probabilística, escolhida por acessibilidade.

A pesquisa foi aplicada via plataforma on-line às empresas pertencentes à indústria de transformação. Basicamente foram contempladas empresas da indústria têxtil (48,7\%), maquinários (31,4\%) e química (12,3\%). As demais indústrias representam $7,6 \%$ da amostra. A amostra final foi de 86 respondentes. O questionário utilizado foi composto por questões fechadas, que deveriam ser respondidas por meio de escala do tipo Likert, com pontuações variando de 1 ("Discordo totalmente") a 5 ("Concordo totalmente"). A Figura 2 apresenta os construtos da pesquisa. 


\begin{tabular}{|c|c|c|}
\hline $\begin{array}{c}\text { Variáveis } \\
\text { Independentes }\end{array}$ & Dimensões & Autores \\
\hline $\begin{array}{l}\text { Envolvimento dos } \\
\text { Fornecedores }\end{array}$ & $\begin{array}{l}\text { 1- Nós envolvemos os principais fornecedores no processo de desenvolvimento de } \\
\text { novos produtos. } \\
2 \text { - Nossos principais fornecedores participam da equipe de desenvolvimento de } \\
\text { novos produtos. } \\
3 \text { - Nossos principais fornecedores têm influência no projeto de novos produtos. } \\
4 \text { - Há um consenso na empresa de que o envolvimento com o fornecedor é } \\
\text { necessário no processo de desenvolvimento de novos produtos. } \\
5 \text { - Nós envolvemos nossos principais fornecedores no planejamento estratégico da } \\
\text { empresa. } \\
6 \text { - Nós temos um planejamento em conjunto com nossos fornecedores sobre } \\
\text { questões específicas. }\end{array}$ & $\begin{array}{l}\text { He et al. (2014); } \\
\text { Wong et al., (2011); } \\
\text { Sun et al., (2010); } \\
\text { Lau et al., (2010). }\end{array}$ \\
\hline $\begin{array}{l}\text { Envolvimento dos } \\
\text { Clientes }\end{array}$ & $\begin{array}{l}1 \text { - No desenvolvimento do conceito do produto nós consideramos as necessidades } \\
\text { de nossos clientes. } \\
2 \text { - Nós visitamos nossos clientes para discutir questões relacionadas ao } \\
\text { desenvolvimento de novos produtos. } \\
3 \text { - Nós buscamos saber como os nossos clientes usam os nossos produtos. } \\
4 \text { - Nossa equipe de desenvolvimento de novos produtos se encontra regularmente } \\
\text { com nossos clientes. }\end{array}$ & $\begin{array}{l}\text { He et al. (2014); } \\
\text { Voss (2012); } \\
\text { Wong et al., (2011); } \\
\text { Stenmark et al., (2011). }\end{array}$ \\
\hline $\begin{array}{c}\text { Variável } \\
\text { Dependente }\end{array}$ & Dimensões & Autores \\
\hline $\begin{array}{l}\text { Capacidade de } \\
\text { Inovação }\end{array}$ & $\begin{array}{l}1 \text { - Nós investimos em pesquisa e desenvolvimento (P\&D) de novos produtos. } \\
2 \text { - Nós buscamos informações e resultados externos ao nosso P\&D. } \\
3 \text { - Nós investimos no aperfeiçoamento das nossas habilidades de aquisição externa } \\
\text { de outros conhecimentos. } \\
4 \text { - Nós investimos na aquisição de máquinas e equipamentos modernos. } \\
5 \text { - Nós investimos na capacitação e treinamento dos nossos colaboradores. } \\
6 \text { - Nós promovemos a introdução de novas tecnologias. }\end{array}$ & $\begin{array}{l}\text { Skippari et al., (2017) } \\
\text { Boly, Morel \& Camargo, } \\
\text { (2014); } \\
\text { Jean et al. (2012); } \\
\text { Fawcett et al., (2012). }\end{array}$ \\
\hline $\begin{array}{c}\text { Variável } \\
\text { Moderadora }\end{array}$ & Dimensões & Autores \\
\hline Confiança & $\begin{array}{l}1 \text { - Nossos parceiros estão preocupados com o nosso bem-estar. } \\
2 \text { - Nossos parceiros consideram como suas decisões/ações nos afetam. } \\
3 \text { - Não podemos confiar nos nossos parceiros. } \\
4 \text { - Nossos parceiros buscam atender nossos interesses. }\end{array}$ & $\begin{array}{l}\text { He et al. (2014); } \\
\text { Yeniyurt et al., (2014); } \\
\text { Kühne et al., (2013); } \\
\text { Handfield \& Bechtel (2002). }\end{array}$ \\
\hline
\end{tabular}

Figura 2: Constructos da pesquisa.

Fonte: Elaborado pelos autores.

Os dados foram tabulados no software Excel e analisados utilizando a técnica da Modelagem de Equações Estruturais com auxílio do software SmartPLS. 


\section{Resultados e discussões}

Apresenta-se nesta seção a análise descritiva da pesquisa, bem como os resultados da análise do modelo de equações estruturais. Na Tabela 1 são apresentados os percentuais de resposta da escala Likert, a média e o desvio padrão para cada dimensão.

Tabela 1: Estatística descritiva da pesquisa.

\begin{tabular}{lcccccccc}
\hline \multirow{2}{*}{ Variáveis } & Dimensões & $\mathbf{1}$ & $\mathbf{2}$ & $\mathbf{3}$ & $\mathbf{4}$ & $\mathbf{5}$ & Média & $\begin{array}{c}\text { Desvio } \\
\text { padrão }\end{array}$ \\
\hline \multirow{5}{*}{ Envolvimento de Clientes } & ENVCLI01 & $1,16 \%$ & $1,16 \%$ & $2,33 \%$ & $13,95 \%$ & $81,40 \%$ & 4,73 & 0,676 \\
& ENVCLI02 & $2,33 \%$ & $5,81 \%$ & $4,65 \%$ & $34,88 \%$ & $52,33 \%$ & 4,29 & 0,969 \\
& ENVCLI03 & $3,49 \%$ & $1,16 \%$ & $3,49 \%$ & $17,44 \%$ & $74,42 \%$ & 4,58 & 0,901 \\
& ENVCLI04 & $1,16 \%$ & $9,30 \%$ & $9,30 \%$ & $45,35 \%$ & $34,88 \%$ & 4,03 & 0,963 \\
\hline \multirow{5}{*}{ Envolvimento de } & ENVFOR01 & $11,63 \%$ & $6,98 \%$ & $10,47 \%$ & $41,86 \%$ & $29,07 \%$ & 3,70 & 1,284 \\
& ENVFOR02 & $26,74 \%$ & $12,79 \%$ & $12,79 \%$ & $34,88 \%$ & $12,79 \%$ & 2,94 & 1,442 \\
& ENVFOR03 & $16,28 \%$ & $15,12 \%$ & $13,95 \%$ & $43,02 \%$ & $11,63 \%$ & 3,19 & 1,297 \\
& ENVFOR04 & $6,98 \%$ & $11,63 \%$ & $18,60 \%$ & $33,72 \%$ & $29,07 \%$ & 3,66 & 1,214 \\
& ENVFOR05 & $38,37 \%$ & $12,79 \%$ & $17,44 \%$ & $20,93 \%$ & $10,47 \%$ & 2,52 & 1,445 \\
& ENVFOR06 & $22,09 \%$ & $16,28 \%$ & $13,95 \%$ & $34,88 \%$ & $12,79 \%$ & 3,00 & 1,389 \\
\hline \multirow{5}{*}{ Capacidade de Inovação 0} & CAPINOV01 & $3,49 \%$ & $17,44 \%$ & $11,63 \%$ & $33,72 \%$ & $33,72 \%$ & 3,77 & 1,195 \\
& CAPINOV02 & $3,49 \%$ & $13,95 \%$ & $17,44 \%$ & $39,53 \%$ & $25,58 \%$ & 3,70 & 1,107 \\
& CAPINOV03 & $4,65 \%$ & $10,47 \%$ & $13,95 \%$ & $45,35 \%$ & $25,58 \%$ & 3,77 & 1,092 \\
& CAPINOV04 & $3,49 \%$ & $4,65 \%$ & $8,14 \%$ & $43,02 \%$ & $40,70 \%$ & 4,13 & 0,992 \\
& CAPINOV05 & $2,33 \%$ & $9,30 \%$ & $15,12 \%$ & $40,70 \%$ & $32,56 \%$ & 3,92 & 1,031 \\
& CAPINOV06 & $2,33 \%$ & $5,81 \%$ & $16,28 \%$ & $47,67 \%$ & $27,91 \%$ & 3,93 & 0,943 \\
\hline
\end{tabular}

Fonte: Dados da pesquisa.

Observa-se, de acordo com a Tabela 1, que a variável envolvimento dos fornecedores não obteve unanimidade das seis dimensões analisadas. As dimensões referentes ao envolvimento dos principais fornecedores no processo de desenvolvimento de novos produtos, a influência dos fornecedores no projeto dos novos produtos e o consenso na empresa de que o envolvimento com o fornecedor é necessário no processo de desenvolvimento de novos produtos foram aquelas mais percebidas pelos respondentes, em que mais de $50 \%$ dos respondentes concordam parcialmente ou concordam totalmente com as questões. As questões que se referem à participação dos fornecedores na equipe de desenvolvimento de novos produtos, o envolvimento dos principais fornecedores no planejamento estratégico da empresa e a questão de possuir um planejamento em conjunto com os fornecedores sobre questões específicas apresentaram mais de neutralidade ou discordância nas respostas obtidas. 
Esses resultados sugerem que o envolvimento do fornecedor no desenvolvimento de novos produtos tem sido considerado como parceria comercial em vez de uma integração entre a manufatura e o fornecedor. Isso se dá porque o envolvimento do fornecedor é pontual para o objetivo específico de desenvolver um produto, não fazendo parte da equipe de DNP e não participando do planejamento de longo prazo da organização de manufatura. Assim, entende-se que esse envolvimento está mais relacionado à obtenção de matéria-prima ou tecnologias que são adequadas ao novo produto do que àqueles voltados para desenvolvimento e competitividade da cadeia.

Em relação à variável envolvimento de clientes, o percentual de concordância é superior a 50\% para todas as quatro dimensões analisadas, sendo elas: no desenvolvimento do conceito do produto considera-se as necessidades dos clientes, visita-se os clientes para discutir questões relacionadas ao desenvolvimento de novos produtos, busca-se saber como os clientes usam os produtos e a equipe de desenvolvimento de novos produtos se encontra regularmente com os clientes.

Nesse tipo de envolvimento, verifica-se uma orientação para o mercado a partir da organização de manufatura. Isso porque os clientes são o elo da cadeia mais próximos ao consumidor final, do qual são extraídas as informações sobre as necessidades, expectativa de qualidade e funcionalidade dos produtos. Visitas frequentes são necessárias para que se entenda e absorva as exigências do mercado, desenvolvendo, assim, produtos mais ajustados às demandas.

No que diz respeito à variável capacidade de inovação, todas as seis dimensões (investe-se em pesquisa e desenvolvimento, P\&D, de novos produtos, busca-se informações e resultados externos ao P\&D, investe-se no aperfeiçoamento das habilidades de aquisição externa de outros conhecimentos, investe-se na aquisição de máquinas e equipamentos modernos, investe-se na capacitação e treinamento dos colaboradores e promove-se a introdução de novas tecnologias) obtiveram também mais de 50\% de concordância.

Dessa forma, pode-se inferir que as organizações de manufatura estão preparadas para ajustar os processos de produção e desenvolver novos produtos em face dos investimentos em recursos que podem alavancar o grau de inovação das organizações. Portanto, a capacidade de inovação pode refletir o grau de flexibilidade das organizações para atendimento às demandas, aumentando, assim, o desempenho operacional.

A variável confiança apresentou em três questões com concordância superior a 50\%, as quais são: os parceiros estão preocupados com o nosso bem-estar; os parceiros consideram como suas decisões/ações nos afetam e os parceiros buscam atender nossos interesses. Apenas a questão "não podemos confiar nos nossos parceiros" não obteve concordância, mas, por se tratar de uma questão de sentido reverso, sugere-se que para as quatro questões houve concordância sobre a confiança nos parceiros. Essas concordâncias refletem que as organizações de manufatura percebem seus clientes e fornecedores como fontes de informações confiáveis, que cumprem os prazos e responsabilidades atribuídas no processo de desenvolvimento de produtos. 
Isso não significa que as organizações confiem em fornecedores e clientes como parceiros no negócio, com responsabilidades compartilhadas em caso de sucesso e insucesso dos produtos ou na busca da competitividade da cadeia. Isso ocorre devido ao grau de concordância percebido nas questões de envolvimento de parceiros no planejamento estratégico da organização.

Na sequência, serão evidenciadas as análises da modelagem de equações estruturais, contemplando os procedimentos: Alpha de Cronbach (AC), Confiabilidade Composta (CR), Variância Média Extraída (AVE), Validade Discriminante e Teste T de Student para cada dimensão.

De acordo com os testes de confiabilidade, os valores obtidos para Alpha de Cronbach e Confiabilidade Composta foram superiores aos parâmetros recomendados pela literatura $(0,6)$. Os valores para Variância Média Extraída também foram superiores aos parâmetros recomendados pela literatura $(0,5)$. A exceção foi a assertiva CONFIA03, que foi retirada do modelo por não atingir a carga fatorial mínima $(0,50)$ recomendada pela literatura (Chin, 2010). Após a retirada da assertiva CONFIA03, os testes foram rodados novamente e os valores se encontram na Tabela 2.

Tabela 2: Testes de confiabilidade.

\begin{tabular}{lccc}
\hline \multicolumn{1}{c}{ Variáveis } & Alpha de Cronbach & Confiabilidade Composta & Variância média extraída \\
\hline 1 Envolvimento de Clientes & 0,814 & 0,877 & 0,642 \\
2 Envolvimento de Fornecedores & 0,862 & 0,896 & 0,592 \\
3 Capacidade de Inovação & 0,882 & 0,911 & 0,630 \\
4 Confiança & 0,869 & 0,919 & 0,791 \\
\hline
\end{tabular}

Fonte: Dados da pesquisa.

A etapa seguinte deu-se pela análise da validade discriminante, segundo critério de Fornell e Larcker (1981) e o critério das cargas cruzadas (Chin, 1998). A Tabela 3 apresenta os resultados da validade discriminante segundo Fornell e Larcker (1981), cujos valores encontrados são suportados pelo critério estabelecido pelos autores. Nessa análise, de acordo com Ringle, Silva e Bido (2014), o valor de cada coluna (em destaque) deverá ser maior do que a correlação entre os constructos.

Tabela 3: Validade discriminante.

\begin{tabular}{lcccc}
\hline \multicolumn{1}{c}{ Variáveis } & $\begin{array}{c}\text { 1 Envolvimento } \\
\text { de Clientes }\end{array}$ & $\begin{array}{c}\text { 2 Envolvimento } \\
\text { de Fornecedores }\end{array}$ & $\begin{array}{c}\text { 3 Capacidade de } \\
\text { Inovação }\end{array}$ & 4 Confiança \\
\hline 1 Envolvimento de Clientes & 0,801 & & \\
\hline Envolvimento de Fornecedores & 0,285 & 0,770 & 0,793 \\
3 Capacidade de Inovação & 0,606 & 0,285 & 0,387 & 0,889 \\
\hline Confiança & 0,269 & 0,381 & 0,80 \\
\hline
\end{tabular}

Fonte: Dados da Pesquisa. 
De acordo com os resultados da validade discriminante pelo critério das cargas cruzadas, contidos na Tabela 4, há validade discriminante entre os constructos, pois as cargas encontradas nos constructos individuais foram maiores do que nos outros, reforçando a posição de Ringle, Silva e Bido (2014).

Tabela 4: Validade discriminante referente às cargas cruzadas.

\begin{tabular}{|c|c|c|c|c|c|}
\hline Variáveis & Dimensões & $\begin{array}{c}1 \text { Envolvimento de } \\
\text { Clientes }\end{array}$ & $\begin{array}{c}2 \text { Envolvimento de } \\
\text { Fornecedores }\end{array}$ & $\begin{array}{l}3 \text { Capacidade de } \\
\text { Inovação }\end{array}$ & 4 Confiança \\
\hline \multirow{4}{*}{$\begin{array}{l}1 \text { Envolvimento de } \\
\text { Clientes }\end{array}$} & ENVCLI01 & 0,827 & 0,228 & 0,562 & 0,292 \\
\hline & ENVCLI02 & 0,848 & 0,146 & 0,502 & 0,162 \\
\hline & ENVCLI03 & 0,741 & 0,144 & 0,428 & 0,165 \\
\hline & ENVCLI04 & 0,784 & 0,411 & 0,431 & 0,232 \\
\hline \multirow{6}{*}{$\begin{array}{l}2 \text { Envolvimento de } \\
\text { Fornecedores }\end{array}$} & ENVFOR01 & 0,261 & 0,814 & 0,281 & 0,342 \\
\hline & ENVFOR02 & 0,287 & 0,816 & 0,205 & 0,252 \\
\hline & ENVFOR03 & 0,176 & 0,772 & 0,176 & 0,274 \\
\hline & ENVFORO4 & 0,223 & 0,830 & 0,235 & 0,254 \\
\hline & ENVFOR05 & 0,175 & 0,736 & 0,229 & 0,357 \\
\hline & ENVFOR06 & 0,176 & 0,633 & 0,153 & 0,264 \\
\hline \multirow{6}{*}{$\begin{array}{l}3 \text { Capacidade de } \\
\text { Inovação }\end{array}$} & CAPIN0V01 & 0,506 & 0,232 & 0,819 & 0,252 \\
\hline & CAPINOV02 & 0,477 & 0,218 & 0,781 & 0,305 \\
\hline & CAPINOV03 & 0,434 & 0,217 & 0,773 & 0,372 \\
\hline & CAPINOV04 & 0,540 & 0,214 & 0,787 & 0,303 \\
\hline & CAPINOV05 & 0,387 & 0,158 & 0,746 & 0,281 \\
\hline & CAPINOV06 & 0,519 & 0,304 & 0,850 & 0,329 \\
\hline \multirow{3}{*}{4 Confiança } & CONFIA01 & 0,229 & 0,359 & 0,364 & 0,931 \\
\hline & CONFIA02 & 0,280 & 0,423 & 0,381 & 0,896 \\
\hline & CONFIA04 & 0,199 & 0,201 & 0,272 & 0,839 \\
\hline
\end{tabular}

Fonte: Dados da Pesquisa.

Iniciando a avaliação do modelo estrutural, tem-se como objetivo avaliar os valores de $\mathrm{R}^{2}, \mathrm{Q}^{2}, \mathrm{~F}^{2}$, Teste T de Student, $p$-valuee, por fim, o coeficiente de caminho. A Tabela 5 apresenta os valores de $R^{2}$ (coeficiente de determinação de Pearson) obtidos pelo modo PLS Algorithm, do $Q^{2}$ (validade preditiva ou indicador de Stone-Geisser) e do $\mathrm{F}^{2}$ (tamanho do efeito ou indicador de Cohen) obtidos pelo modo Blindfolding.

Tabela 5: Valores de $\mathrm{R}^{2}, \mathrm{Q}^{2}$ e $\mathrm{f}^{2}$.

\begin{tabular}{lccc}
\hline \multicolumn{1}{c}{ Variáveis } & $\mathbf{R}^{2}$ & $\mathbf{Q}^{2}$ & $\mathrm{~F}^{2}$ \\
\hline 1 Envolvimento de Clientes & & & 0,389 \\
2 Envolvimento de Fornecedores & & & 0,420 \\
3 Capacidade de Inovação & 0,424 & 0,234 & 0,462 \\
4 Confiança & & & 0,533 \\
\hline
\end{tabular}

Fonte: Dados da pesquisa. 
Finalizando a análise estatística do modelo de equações estruturais, a análise dos valores do Teste T de Student e $p$-value entre os constructos foi realizada pelo modo Bootstrapping do software SmartPLS. A literatura indica para o teste T de Student valores $\geq 1,96$ e para o $p$-value valores $<0,05$. A Tabela 6 apresenta os resultados relativos ao Teste T de Student e o p-value.

Tabela 6: Teste T de Student e p-value nas relações entre os constructos.

\begin{tabular}{clccc}
\hline Hipóteses & \multicolumn{1}{c}{ Relação entre os constructos da pesquisa } & $\begin{array}{c}\text { Coeficiente } \\
\text { de Caminho }\end{array}$ & $\begin{array}{c}\text { Teste T de } \\
\text { Student }\end{array}$ & p-value \\
\hline H1 & 1 Envolvimento de Clientes $\rightarrow$ 3 Capacidade de inovação & 0,520 & 5,045 & 0,000 \\
H2 & 2 Envolvimento de Fornecedores $\rightarrow$ 3 Capacidade de inovação & 0,046 & 0,462 & 0,644 \\
H3a & $\begin{array}{l}\text { 5 Envolvimento de Clientes Moderado pela Confiança } \rightarrow \text { 3 Capacidade de } \\
\text { inovação }\end{array}$ & 0,015 & 0,173 & 0,862 \\
H3b & $\begin{array}{l}\text { 6 Envolvimento de Fornecedores Moderado pela Confiança } \rightarrow \text { 3 Capacidade } \\
\text { de inovação }\end{array}$ & 0,003 & 0,035 & 0,972 \\
\hline
\end{tabular}

Fonte: Dados da pesquisa.

De acordo com a Tabela 6 é possível verificar que apenas uma relação se manteve entre os parâmetros indicados pela literatura: Envolvimento de Clientes $\rightarrow$ Capacidade de Inovação. Três relações não se mantiveram entre os parâmetros indicados pela literatura: Envolvimento de Fornecedores $\rightarrow$ Capacidade de Inovação; Envolvimento de Clientes Moderado pela Confiança $\rightarrow$ Capacidade de Inovação e Envolvimento de Fornecedores Moderado pela Confiança $\rightarrow$ Capacidade de Inovação. Sendo assim, a não significância dessas foram determinadas relações e, consequentemente, o não suporte das hipóteses $\mathrm{H}_{2}, \mathrm{H}_{3}$ a e $\mathrm{H}_{3 b}$.

Conforme exposto na Tabela 6, o Envolvimento de Clientes influencia 52\% a Capacidade de Inovação Organizacional. Esta afirmação é validada pelos resultados do Teste $T$ de Student de 5,045 e do p-value de 0,000, dentro do recomendado pela literatura. Esse resultado está alinhado com os achados de Jean et al. (2012), Stenmark et al (2011), Johnson e Filippini (2009). Szeto (2000) sugere que a capacidade de inovação pode ser definida como os recursos necessários que a empresa possui para transformar oportunidades em novos produtos e processos, atendendo às necessidades dos clientes. Boly et al., (2014) pontuam que em uma revisão da literatura identificaram muitas sugestões para medir a capacidade de inovação organizacional, sendo a colaboração com os clientes uma dessas variáveis.

A influência do envolvimento do cliente na capacidade de inovação pode estar associada à orientação para o mercado da organização focal percebida por meio da estatística descritiva. Assim, com base nas informações obtidas nas interações com clientes, são feitos investimentos na capacidade da organização de inovar, que podem influenciar a flexibilidade dos meios de produção e a inovação dos produtos.

Com influência de 4,6\% na Capacidade de Inovação, o Envolvimento de Fornecedores não apresentou resultado significativo nesta pesquisa. O resultado difere dos achados de He et al. (2014), Sun et al., (2010), Lau et al., (2010) e Handfield et al. (1999), que afirmam que o envolvimento dos fornecedores aumenta o desempenho dos novos produtos, da capacidade de inovação e da qualidade, trazendo vantagens competitivas para a organização. 
No contexto desta pesquisa, a não relação entre envolvimento do fornecedor e capacidade de inovação pode ser evidenciada pelo grau de comprometimento entre as partes para o DNP. De acordo com a descrição dos resultados, o fornecedor é visto como um provedor de matéria-prima e tecnologias, que participa do processo de desenvolvimento de produtos de forma pontual, não se integrando às questões estratégicas relacionadas à competitividade da cadeia. Este envolvimento, de certa forma superficial, pode ser decorrente da desconfiança das organizações de manufatura em compartilhar informações que possam ser disseminadas pelos fornecedores aos concorrentes. Ressalta-se que a confiança verificada inicialmente na estatística descritiva é referente ao fornecedor como parceiro comercial, mas não como parceiro no negócio que visa buscar a competitividade da cadeia.

A Confiança como moderadora do Envolvimento de Clientes e Fornecedores na Capacidade de Inovação também não apresentou resultado significativo nesta pesquisa. Tanto o Teste $T$ de Student como o $p$-value não apresentaram resultados indicados pela literatura. Esses resultados contradizem os achados de Kühne et al., (2013), que sugerem que a confiança é um fator importante para a melhoria da capacidade de inovação das organizações. Contudo, para Molina-Morales et al., (2011), as empresas que investem demais na confiança, assumindo riscos desnecessários, podem ter efeitos negativos na capacidade de inovação.

Assim, pode-se dizer que, nesta pesquisa, a confiança não é um fator que leva o envolvimento de clientes e fornecedores a influenciar o aumento da capacidade de inovação. A orientação para o mercado verificada no envolvimento do cliente no DNP é condição para que a organização aumente seus investimentos para inovar, mas isso independe no nível de confiança estabelecido entre a manufatura-cliente. No caso do fornecedor, como o envolvimento é comercial e não influencia nos investimentos em capacidade de inovação, ter ou não confiança não altera a relação entre essas variáveis.

Isso ocorre porque a confiança estabelecida entre a manufatura, clientes e fornecedores não está relacionada ao comprometimento destes enquanto integração de cadeia para planejamentos futuros ou busca da competitividade, mas para ações específicas do desenvolvimento do produto, as quais não foram percebidas como suficientes para aumentar a capacidade de inovação.

\section{Considerações finais}

Este estudo teve como objetivo identificar se a integração de clientes e fornecedores influenciam a capacidade de inovação organizacional. Ao explorar a integração da cadeia de suprimentos e a capacidade de inovação destacam-se os processos de alianças, enfatizados pela TDR. Lii e Kuo (2016) reforçam que a essência da TDR é que quando uma organização não tem ou não pode gerar os recursos necessários, ela pode entrar em uma relação de troca com outras organizações. 
Os resultados desta pesquisa oferecem novos insights sobre a influência da integração externa da cadeia de suprimentos na capacidade de inovação organizacional. A pesquisa demonstrou que a integração externa com os clientes está positivamente relacionada com a capacidade de inovação organizacional. Os resultados também indicaram que o envolvimento dos fornecedores e o envolvimento de clientes e fornecedores moderado pela confiança não exercem influência significante sobre a capacidade de inovação.

Esses achados contribuem para a literatura referente à integração da cadeia de suprimentos e capacidade de inovação, uma vez que grande parte dos estudos anteriores tem focado na ICS e o desempenho organizacional (Lii \& Kuo, 2016, Wong et al., 2013; Lau et al., 2010; Kim, 2009). E, de acordo com Liu et al. (2013), Prajogo e Olhager (2012) e Yu, Jacobs, Chavez \& Feng, (2017), a literatura reconhece a associação entre a integração da cadeia de suprimentos e a capacidade de inovação, no entanto, há relativamente pouca evidência empírica da natureza exata dessa relação.

Em relação à teoria utilizada neste estudo, verificou-se que a dependência de recursos para gerar capacidade de inovação vem basicamente de clientes, uma vez que as organizações estudadas consideram as informações de mercado mais relevantes do que as informações de fornecedores para gerar capacidade de inovação. Conforme os resultados, os recursos se resumem às informações de mercado e não necessariamente ao ganho potencial proveniente da inclusão de parceiros no planejamento da organização, que pode gerar vantagem competitiva, conforme preconizado pela Teoria da Dependência de Recursos. Assim, verificou-se que o nível de envolvimento com fornecedores para desenvolvimento de novos produtos (DNP) não é complexo a ponto de gerar capacidade de inovação.

Referente às limitações da pesquisa, pode-se destacar a amostra de organizações de vários setores e porte diferentes. Outra limitação da investigação diz respeito ao fato de que os dados são essencialmente transversais. Como pesquisas futuras sugere-se a aplicação do questionário em organizações do mesmo setor e porte, bem como a realização de um estudo longitudinal. Skippari et al., (2017) reforçam que as relações entre os membros da cadeia de suprimentos mudam e evoluem ao longo do tempo, sendo assim, é pertinente que estudos futuros investiguem essas evoluções. Além disso, sugere-se ampliar o espectro da pesquisa, tratando do comportamento organizacional e das relações de poder na cadeia de suprimentos, bem como abordar aspectos da governança corporativa na demanda dos parceiros da cadeia para sobrevivência e competitividade. 


\section{Referências}

Abdolmaleki, K., \& Ahmadian, S. (2016). The relationship between product characteristics, customer and supplier involvement and new product development. Procedia Economics and Finance, 36, 147-156.

Ayoub, H. F., Abdallah, A. B., \& Suifan, T. S. (2017). The effect of supply chain integration on technical innovation in Jordan: the mediating role of knowledge management. Benchmarking: An International Journal, 24(3), 594-616.

Birasnav, M., Albufalasa, M., \& Bader, Y. (2013). The role of transformational leadership and knowledge management processes on predicting product and process innovation: An empirical study developed in Kingdom of Bahrain. Tékhne, 11(2), 64-75.

Boly, V., Morel, L., \& Camargo, M. (2014). Evaluating innovative processes in French firms: Methodological proposition for firm innovation capacity evaluation. Research Policy, 43(3), 608-622.

Bornia, A. C., \& Lorandi, J. A. (2016). O processo de desenvolvimento de produtos compartilhados na cadeia de suprimentos. Revista da FAE, 11(2), 35-50.

Bunduchi, R. (2013). Trust, partner selection and innovation outcome in collaborative new product development. Production planning \& control, 24(2-3), 145-157.

Buvik, A., \& Grønhaug, K. (2000). Inter-firm dependence, environmental uncertainty and vertical co-ordination in industrial buyer-seller relationships. Omega, 28(4), 445454.

Chang, L., Ouzrout, Y., Nongaillard, A., Bouras, A., \& Jiliu, Z. (2014). Multi-criteria decision making based on trust and reputation in supply chain. International Journal of Production Economics, 147(Part B), 362-372.

Chen, J. V., Yen, D. C., Rajkumar, T. M., \& Tomochko, N. A. (2011). The antecedent factors on trust and commitment in supply chain relationships. Computer Standards \& Interfaces, 33(3), 262-270.

Chin, W. W. (1998). The partial least squares approach to structural equation modeling. Modern methods for business research, 295(2), 295-336.

Chin W.W. (2010) Bootstrap Cross-Validation Indices for PLS Path Model Assessment. In: Esposito Vinzi V., Chin W., Henseler J., Wang H. (eds) Handbook of Partial Least Squares. Springer, Berlin, Heidelberg: Springer Handbooks of Computational Statistics.

Drees, J. M., \& Heugens, P. P. (2013). Synthesizing and extending resource dependence theory: A meta-analysis. Journal of Management, 39(6), 1666-1698.

Fawcett, S. E., Jones, S. L., \& Fawcett, A. M. (2012). Supply chain trust: The catalyst for collaborative innovation. Business Horizons, 55(2), 163-178.

Fornell, C., \& Larcker, D. F. (1981). Evaluating structural equation models with unobservable variables and measurement error. Journal of marketing research, 18(1), 39-50.

Forsman, H. (2011). Innovation capacity and innovation development in small enterprises. A comparison between the manufacturing and service sectors. Research Policy, 40(5), 739-750. 
Gonzalez-Zapatero, C., Gonzalez-Benito, J., \& Lannelongue, G. (2017). Understanding how the functional integration of purchasing and marketing accelerates new product development. International Journal of Production Economics, 193, 770-780.

Handfield, R. B., Ragatz, G. L., Petersen, K. J., \& Monczka, R. M. (1999). Involving suppliers in new product development. California management review, 42(1), 59-82. Handfield, R. B., \& Bechtel, C. (2002). The role of trust and relationship structure in improving supply chain responsiveness. Industrial marketing management, 31(4), 367-382.

He, Y., Lai, K. K., Sun, H., \& Chen, Y. (2014). The impact of supplier integration on customer integration and new product performance: the mediating role of manufacturing flexibility under trust theory. International Journal of Production Economics, 147, 260-270.

Jean, R. J., Kim, D., \& Sinkovics, R. R. (2012). Drivers and performance outcomes of supplier innovation generation in customer-supplier relationships: The role of power - dependence. Decision Sciences, 43(6), 1003-1038.

Johnson, W. H., \& Filippini, R. (2009). Internal vs. external collaboration: What works. Research-Technology Management, 52(3), 15-17.

Kim, S. W. (2009). An investigation on the direct and indirect effect of supply chain integration on firm performance. International Journal of Production Economics, 119(2), 328-346.

Kühne, B., Gellynck, X., \& Weaver, R. D. (2013). The influence of relationship quality on the innovation capacity in traditional food chains. Supply Chain Management: An International Journal, 18(1), 52-65.

Langerak, F., \& Hultink, E. J. (2008). The effect of new product development acceleration approaches on development speed: A case study. Journal of Engineering and Technology Management, 25(3), 157-167.

Lau, A. K., Yam, R. C., \& Tang, E. P. (2010). Supply chain integration and product modularity: An empirical study of product performance for selected Hong Kong manufacturing industries. International Journal of Operations \& Production Management, 30(1), 20-56.

Lii, P., \& Kuo, F. I. (2016). Innovation-oriented supply chain integration for combined competitiveness and firm performance. International Journal of Production Economics, 174, 142-155.

Liu, H., Ke, W., Kee Wei, K., \& Hua, Z. (2013). Effects of supply chain integration and market orientation on firm performance: Evidence from China. International Journal of Operations \& Production Management, 33(3), 322-346.

MANUAL, D. O. (2005). Diretrizes para coleta e interpretação de dados sobre inovação. (Tradução de Flávia Gouveia). Publicação conjunta da OCDE e Eurostat. Versão Brasileira: Financiadora de Estudos e Projetos (Finep).

Martins, G. S. (2013). A medida de confiar é confiar sem medida? Estudo sobre o efeito da confiança na criação e na captura de valor na relação compradorfornecedor. Tese de doutorado, Fundação Getúlio Vargas, São Paulo, SP, Brasil.

Meznar, M. B., \& Nigh, D. (1995). Buffer or bridge? Environmental and organizational determinants of public affairs activities in American firms. Academy of Management Journal, 38(4), 975-996. 
Molina-Morales, F. X., Martínez-Fernández, M. T., \& Torlò, V. J. (2011). The dark side of trust: The benefits, costs and optimal levels of trust for innovation performance. Long Range Planning, 44(2), 118-133.

Ojha, D., Shockley, J., \& Acharya, C. (2016). Supply chain organizational infrastructure for promoting entrepreneurial emphasis and innovativeness: The role of trust and learning. International Journal of Production Economics, 179, 212-227.

Pfeffer, J. (1987). A resource dependence perspective on intercorporate relations. In: Mizruchi, M. S.; Schwartz, M. (Eds.). Intercorporate Relations. New York: Cambridge University Press.

Pfeffer, J., \& Salancik, G. R. (2003). The external control of organizations: A resource dependence perspective. Califórnia: Stanford University Press.

Prajogo, D., \& Olhager, J. (2012). Supply chain integration and performance: The effects of long-term relationships, information technology and sharing, and logistics integration. International Journal of Production Economics, 135(1), 514-522.

Ringle, C. M., Silva, D., \& Bido, D. D. S. (2014). Modelagem de equações estruturais com utilização do SmartPLS. REMark, 13(2), 54.

Schiele, H., Ellis, S. C., Eßig, M., Henke, J. W., \& Kull, T. J. (2015). Managing supplier satisfaction: Social capital and resource dependence frameworks. Australasian Marketing Journal (AMJ), 23(2), 132-138.

Shou, Y., Li, Y., Park, Y. W., \& Kang, M. (2017). The impact of product complexity and variety on supply chain integration. International Journal of Physical Distribution \& Logistics Management, 47(4), 297-317.

Skippari, M., Laukkanen, M., \& Salo, J. (2017). Cognitive barriers to collaborative innovation generation in supply chain relationships. Industrial Marketing Management, 62, 108-117.

Stenmark, P., Tinnsten, M., \& Wiklund, H. (2011). Customer involvement in product development: Experiences from Scandinavian outdoor companies. Procedia Engineering, 13(2011),538-543.

Sun, H., Yau, H. K., Suen, M., \& Kwok, E. (2010). The simultaneous impact of supplier and customer involvement on new product performance. Journal of technology management \& innovation, 5(4), 70-82.

Szeto, E. (2000). Innovation capacity: working towards a mechanism for improving innovation within an inter-organizational network. The TQM Magazine, 12(2), 149158.

Van de Ven, A. H. (1986). Central problems in the management of innovation. Management science, 32(5), 590-607.

Vijayasarathy, L. R. (2010). Supply integration: an investigation of its multidimensionality and relational antecedents. International Journal of Production Economics, 124(2), 489-505.

Voss, M. (2012). Impact of customer integration on project portfolio management and its success-Developing a conceptual framework. International Journal of Project Management, 30(5), 567-581.

Wong, C. Y., Boon-Itt, S., \& Wong, C. W. (2011). The contingency effects of environmental uncertainty on the relationship between supply chain integration and operational performance. Journal of Operations management, 29(6), 604-615. 
Wong, C. W., Wong, C. Y., \& Boon-itt, S. (2013). The combined effects of internal and external supply chain integration on product innovation. International Journal of Production Economics, 146(2), 566-574.

Yeniyurt, S., Henke, J. W., \& Yalcinkaya, G. (2014). A longitudinal analysis of supplier involvement in buyers' new product development: working relations, interdependence, co-innovation, and performance outcomes. Journal of the Academy of Marketing Science, 42(3), 291-308.

Yu, W., Jacobs, M. A., Chavez, R., \& Feng, M. (2017). The impacts of IT capability and marketing capability on supply chain integration: a resource-based perspective. International Journal of Production Research, 55(14), 4196-4211.

Zhao, X., Huo, B., Flynn, B. B., \& Yeung, J. H. Y. (2008). The impact of power and relationship commitment on the integration between manufacturers and customers in a supply chain. Journal of Operations Management, 26(3), 368-388. 